\title{
A flora apícola de uma área restrita de dunas litorâneas, Abaeté, Salvador, Bahia
}

\author{
BLANDINA FELIPE VIANA ${ }^{1,4}$, FABIANA OLIVEIRA DA SILVA² e ASTRID DE M.P. KLEINERT ${ }^{3}$
}

(recebido: 9 de junho de 2004; aceito: 29 de dezembro de 2005)

\begin{abstract}
The bee flora of the sea coastal sand dunes of Abaeté, Salvador, Bahia State, Brazil). The bee plant community in a fragment of 8.2 ha in the Environmental ProtectionArea of "Lagoas e Dunas de Abaeté", in Salvador, Bahia, Brazil (1256' S $138^{\circ} 21^{\prime}$ W) was investigated. Between January and December 1996, three times a month, blooming plants were sampled. Floral traits such as size, shape, symmetry, sexuality, anther dehiscence, and colour; floral rewards and flowering period were recorded for each species. Restinga, a sand dune scrub is the vegetation type, formed mainly by shrubs and subshrubs. Ninety-seven plant species were observed, Fabaceae was the richest family in number of species. Bees visited 66 species but concentrated their visits in 12 species (79.4\% of total of individuals). Waltheria cinerescens St. Hilaire and Byrsonima microphylla A. Juss. were the most abundant species in the area. Floral resources were available for the bees during the whole year but the number of flowers was greater at the low rainfall season. In general, flowers remained open during the whole day; most of them were monoclinous (89\% of species), small, tubular, pink-purple (32\%) and cream (31\%), actinomorphic (63\%) and arranged in inflorescences. Most of the species are mellitophilous (85\%), it means that bees are probably the main pollen vector in this ecosystem.
\end{abstract}

Key words - Apoidea, bee plants, restinga, sand dunes

RESUMO -(A flora apícola de uma área restrita de dunas litorâneas, Abaeté, Salvador, Bahia). A comunidade de plantas visitadas por abelhas foi estudada em um fragmento de 8,2 ha na Área de Proteção Ambiental das Lagoas e Dunas de Abaeté, Salvador, Bahia (1256' S e 38²1' W). Entre janeiro e dezembro de 1996, três vezes ao mês, as plantas floridas eram amostradas, registrando-se para cada espécie o período de floração, hábito e características florais como: cor, forma, sexualidade, simetria, deiscência das anteras e recurso oferecido ao visitante. O tipo de vegetação local é a restinga, composta principalmente por arbustos e subarbustos. Foram observadas 97 espécies vegetais e a família Fabaceae foi a mais rica em número de espécies. Das espécies observadas, 66 foram visitadas por abelhas, sendo que 12 delas foram predominantemente visitadas (79,4\% do total de indivíduos). Waltheria cinerescens St. Hilaire e Byrsonima microphylla A. Juss. foram as espécies mais abundantes. Os recursos florais estiveram disponíveis ao longo de todo o ano, havendo maior produção de flores nos meses de menor precipitação. A maioria das flores esteve aberta durante todo o dia. Predominaram flores actinomorfas (63\%), monóclinas (89\%), pequenas, tubulares e reunidas em inflorescências, cujas cores mais freqüentes são lilás (32\%) e creme (31\%). A maioria era melitófila (85\%), significando que as abelhas são, provavelmente, os principais responsáveis pela reprodução sexual das espécies vegetais nessas dunas.

Palavras-chave - Apoidea, dunas litorâneas, plantas apícolas, restinga

\section{Introdução}

A composição florística das comunidades de restinga no litoral brasileiro, principalmente das regiões sul e sudeste, foi tratada em diversos estudos (Rawistscher 1944, Magnanini 1954, Andrade 1968, Pfadenhauer 1978, Bressolin 1979, Araújo \& Henriques 1984, Cordazzo \& Seeliger 1987, Pereira \& Gomes 1994,

1. Universidade Federal da Bahia, Instituto de Biologia, Departamento de Zoologia, Laboratório de Biologia e Ecologia de Abelhas (Labea). Rua Barão de Geremoabo s/n, Campus Universitário de Ondina, 40170-110 Salvador, BA, Brasil.

2. Faculdade Tecnologia e Ciências (FTC). Av. Luís Viana Filho, 8812, Paralela, 41820-785 Salvador, BA, Brasil.

3. Universidade de São Paulo, Departamento de Ecologia, Instituto de Biociências. Rua do Matão, travessa 14, 321, Cidade Universitária, 05508-900 São Paulo, SP, Brasil.
Silva et al. 1994, Sugiyama \& Mantovani 1994). Em relação às restingas no litoral nordestino há poucas informações (Silva 1972, Pinto et al. 1984).

As informações pioneiras sobre a flora das dunas de Abaeté, disponíveis na literatura, podem ser encontradas em Torrend (1938) e Seabra (1949). Mais recentemente, Britto et al. (1993) identificaram nessa área 410 espécies vegetais pertencentes a 283 gêneros de 88 famílias. Esses trabalhos revelaram a elevada diversidade taxonômica e o número expressivo de espécies endêmicas da flora fanerogâmica das dunas de Abaeté (Morawetz 1983, Plowman 1983), contrapondo-se a informações levantadas em ecossistemas similares de restinga (Araújo \& Lacerda 1987, Sugiyama \& Mantovani 1994).

Nenhum dos estudos acima menciona os visitantes florais e as síndromes de polinização das espécies 
vegetais presentes nas áreas de dunas. Gottsberger et al. (1988), em pesquisa realizada nas dunas litorâneas de São Luís, Maranhão, destacaram a importância dos insetos, em particular das abelhas, na polinização das espécies daquele ambiente. Nove das dez espécies de plantas estudadas por eles eram melitófilas, ou seja, suas flores eram visitadas por Hymenoptera - principalmente abelhas. Muitas vezes, estas abelhas são seus polinizadores, portanto visitantes responsáveis pela reprodução sexual dessas plantas, ao contrário do que ocorre com a vegetação das dunas nas regiões temperadas, onde há uma grande proporção de espécies anemófilas e raras espécies entomófilas (Proctor et al. 1996).

O conhecimento da estrutura da comunidade de plantas visitadas por abelhas nas dunas de Abaeté visa contribuir para o entendimento das interações entre abelhas e flores, que pode fornecer subsídios para os estudos sobre a ecologia da polinização das espécies melitófilas nesse ambiente. Os objetivos específicos deste trabalho foram: a) identificar as espécies visitadas por abelhas, destacando aquelas predominantemente visitadas nas dunas de Abaeté; b) caracterizar as plantas apícolas quanto ao hábito, recursos oferecidos aos visitantes e morfologia floral; c) determinar a fenologia floral durante o período amostral e estimar a quantidade de recursos (número de flores) disponíveis para as abelhas na área de estudos; d) analisar comparativamente a flora apícola das dunas em relação a levantamentos realizados em ecossistemas similares.

\section{Material e métodos}

A vegetação predominante na Área de Proteção Ambiental (APA) das Lagoas e Dunas de Abaeté é a restinga, isolada por matas circundantes. A flora apícola foi amostrada em uma área restrita ( 8,2 ha). A coleta das abelhas e das plantas floridas foi realizada entre janeiro e dezembro de 1996, em intervalos de dez dias.

As abelhas em visita às flores foram coletadas pelo método de varredura, de acordo com Sakagami et al. (1967). Dois coletores percorreram a área, seguindo a mancha de vegetação em direções opostas, entre $06 \mathrm{~h} 00$ e $17 \mathrm{~h} 00$. A cada volta no transecto, as abelhas foram amostradas nas plantas individuais durante cinco minutos. Para calcular a abundância relativa (\%) dos visitantes, nas espécies vegetais predominantemente visitadas, e seus limites de confiança, foi utilizado o método de probabilidade de Katoet al. (1952 apud Laroca 1995). As abelhas foram depositadas na coleção do Laboratório de Biologia e Ecologia de Abelhas (Labea), Instituto de Biologia, Universidade Federal da Bahia. A fauna de Apoidea visitante das flores será listada e discutida em outra publicação.

As espécies vegetais foram identificadas e depositadas nos herbários HRB (IBGE-Salvador), HUEFS (Universidade Estadual de Feira de Santana, Feira de Santana) e ALCB (Universidade Federal da Bahia, Salvador). A organização das famílias seguiu o sistema de Cronquist (1981). A determinação das densidades das plantas apícolas de portes arbóreo, arbustivo e subarbustivo, na área estudada, foi realizada pelo método do quadrante centrado descrito por Greig-Smith (1983). A densidade das herbáceas não foi medida neste estudo.

A similaridade florística entre as dunas de Abaeté e outras comunidades similares onde levantamentos sobre a flora e fauna apícola empregaram o método de Sakagami et al. (1967), foi comparada pelo índice de Sørensen (Southwood 1980). Esse índice considera dados binários (presença/ausência de espécies na amostra), e é dado pela fórmula $\mathrm{CA}=\left[2 \mathrm{c}(\mathrm{a}+\mathrm{b})^{-1}\right] 100$, onde CA é o coeficiente de associação, c é o número de espécies comuns às duas amostras ( 1 e 2), a é o número de espécies da amostra 1 e b o número de espécies da amostra 2.

A amplitude da floração individual, ao longo do período de amostragem, foi avaliada pela contagem do número máximo de flores produzidas por uma dada planta, considerando-se quatro classes de amplitude conforme Newstrom et al. (1993, 1994): ausente, leve, intermediária e intensa. Neste trabalho, porém, a classificação foi empregada para detectar variações em curto prazo, na produção de flores das espécies apícola e acrescentada a categoria intermediária, devido à grande variabilidade existente com relação ao número de flores produzidas por indivíduo.

A quantidade de flores ou de inflorescências disponíveis, em cada espécie vegetal (intensidade de floração) foi avaliada. Em espécies com flores individuais, a produção de flores foi estimada multiplicando-se o número total de indivíduos pelo número médio de flores por indivíduo. Em espécies com inflorescências, contou-se o número médio de flores por inflorescência, e em seguida, o número médio de inflorescências por indivíduo foi multiplicado pelo número total de indivíduos. Em espécies de porte arbustivo e arbóreo foram contados: o número médio de flores (ou número médio de flores/inflorescências) por galho foi multiplicado pelo número médio de galhos por indivíduo; em seguida, o número médio de flores obtido por indivíduo foi multiplicado pelo número total de indivíduos de cada espécie.

As flores foram caracterizadas quanto à cor (considerando-se a cor predominante), simetria da corola, tipo de recurso, sexualidade e, quando possível, o horário de antese. As observações foram feitas em campo, com o auxílio de uma lupa de bolso e, no caso de flores diminutas, em laboratório, complementadas com dados da literatura.

\section{Resultados}

Em Abaeté, 97 espécies vegetais foram observadas. Dentre essas, 66 espécies, abrangendo 55 gêneros e 39 famílias, foram visitadas por abelhas 
(tabela 1). Quando comparada a outros levantamentos em ecossistemas similares, utilizando o método de varredura, a flora apícola de Abaeté apresentou baixa similaridade em nível especifico porém, em nível genérico, a similaridade mostrou-se mais elevada (tabela 2).

As famílias mais importantes quanto ao número de espécies foram Fabaceae (7), Rubiaceae (5), Asteraceae (5), Myrtaceae (4) e Malpighiaceae (4), enquanto a maioria das famílias foi representada por uma espécie. Oito gêneros destacaram-se quanto à diversidade específica Byrsonima (com três espécies) e Lepidaploa, Protium, Centrosema, Struthanthus,
Myrcia, Borreria e Coccoloba (com duas espécies cada).

As espécies com hábito arbustivo predominaram (43\%), seguidas pelas subarbustivas (30\%), herbáceas $(18 \%)$ e trepadeiras (9\%). As três espécies commaior valor de densidade foram Waltheria cinerascens $(23$ ind ha $\left.{ }^{-1}\right)$, Byrsonima microphylla (17 ind $\left.\mathrm{ha}^{-1}\right)$, Lepidaploa arenaria $\left(8 \mathrm{ind} \mathrm{ha}^{-1}\right)$ (tabela 1). Não foi quantificada a densidade de plantas herbáceas, rastejantes e forrações, mas observou-se uma grande concentração das espécies Chamaecrista ramosa, Krameria bahiana, Euphorbia gymnnoclada e Cuphea brachiata.

Tabela 1. Espécies vegetais visitadas por abelhas nas dunas de Abaeté, Salvador, Bahia, Brasil. A abreviação na coluna código substituirá o nome da espécie nas demais tabelas. O hábito das espécies ( $\operatorname{arb}=$ arbustivo; arv = arbóreo; herb = herbáceo; herbhemip = herbáceo hemiparasita; $1 \mathrm{i}=$ liana; subarb = subarbustivo; trep = trepadeira). A densidade $\left(\right.$ ind ha $\left.{ }^{-1}\right)$ das $_{\text {espécies }}$ arbustivas e arbóreas foi obtida pelo método dos quadrantes centrados (Greig-Smith 1983).

Table 1. Plant species visited by bees in the sea coastal sand dunes of Abaeté, Salvador, Bahia, Brazil. Abbreviation on code column will replace species name in the other tables. Habit $(\operatorname{arb}=$ shrub; arv $=$ tree; herb $=$ herbaceous; herb-hemip $=$ hemiparasite herbaceous; li = liana; subarb = subshrub; trep = climber). Density (ind ha ${ }^{-1}$ ) of tree and shrub species was obtained by the point-centered quarter method (Greg-Smith 1983).

\begin{tabular}{|c|c|c|c|c|}
\hline Família/Espécie & Cód. & "Voucher" & Hábito & Ind ha ${ }^{-1}$ \\
\hline \multicolumn{5}{|l|}{ ANACARDIACEAE } \\
\hline Tapirira guianensis Aubl. & $\mathrm{Tg}$ & 33383 & arv & \\
\hline \multicolumn{5}{|l|}{ APOCYNACEAE } \\
\hline Mandevilla funiformis (Vell.) K. Schum. & Mf & 33374 & trep & \\
\hline \multicolumn{5}{|l|}{ ARACEAE } \\
\hline Anthurium longipes N.E. Br. & Al & 33367 & herb & \\
\hline \multicolumn{5}{|l|}{ ARECACEAE } \\
\hline Allagoptera brevicalyx Moraes & Abr & 33430 & arb & \\
\hline \multicolumn{5}{|l|}{ ASCLEPIADACEAE } \\
\hline Ditassa blanchetii Decne. & $\mathrm{Db}$ & 33423 & subarb & \\
\hline \multicolumn{5}{|l|}{ ASTERACEAE } \\
\hline Prolobus nitidus (Baker) R.M. King \& H. Rob. & $\mathrm{Cp}$ & 33378 & subarb & 1,66 \\
\hline Litothamus nitidus (DC.) W.C. Holmes & Ln & 33362 & arb & 1,11 \\
\hline Stilpnopappus scaposus DC. & Ss & 33428 & herb & \\
\hline Lepidaploa arenaria (Mart. ex DC.) H. Rob. & $\mathrm{La}$ & 33381 & subarb & 8,55 \\
\hline L. edmundoi (G.M. Barroso) H. Rob. & Le & 33437 & subarb & \\
\hline \multicolumn{5}{|l|}{ BIGNONIACEAE } \\
\hline Tabebuia elliptica (DC.) Sandwith & $\mathrm{Te}$ & 33400 & arb & \\
\hline \multicolumn{5}{|l|}{ BROMELIACEAE } \\
\hline Aechmea sp. & $\mathrm{Ae}$ & 33451 & herb & \\
\hline \multicolumn{5}{|l|}{ BURSERACEAE } \\
\hline Protium bahianum Daly & $\mathrm{Pb}$ & 33401 & arv & 1,11 \\
\hline P. icicariba (DC.) Marchant & $\mathrm{Pi}$ & 33385 & arv & 1,66 \\
\hline \multicolumn{5}{|l|}{ CAESALPINIACEAE } \\
\hline Chamaecrista ramosa var. ramosa (Vogel) H.S. Irwin \& Barneby & $\mathrm{Cr}$ & 33370 & subarb & \\
\hline \multicolumn{5}{|l|}{ CAPPARACEAE } \\
\hline Dactylaena microphylla Eichler & $\mathrm{Dm}$ & 33368 & subarb & $\begin{array}{c}0,55 \\
\text { continua }\end{array}$ \\
\hline
\end{tabular}


continuação

\begin{tabular}{|c|c|c|c|c|}
\hline Família/Espécie & Cód. & "Voucher" & Hábito & Ind ha $\mathrm{h}^{-1}$ \\
\hline \multicolumn{5}{|l|}{ CLUSIACEAE } \\
\hline Kielmeyera argentea Choisy & $\mathrm{Ka}$ & 33399 & arb & 0,55 \\
\hline \multicolumn{5}{|l|}{ CONVOLVULACEAE } \\
\hline Evolvulus maximiliani Mart. ex Choisy & Em & 33363 & subarb & \\
\hline \multicolumn{5}{|l|}{ ERICACEAE } \\
\hline Agarista revoluta (Spreng.) Hook. f. ex Nied. & $\mathrm{Ar}$ & 33417 & arb & 7,19 \\
\hline \multicolumn{5}{|l|}{ EUPHORBIACEAE } \\
\hline Euphorbia gymnoclada Boiss. & $\mathrm{Eg}$ & 33389 & herb & \\
\hline \multicolumn{5}{|l|}{ FABACEAE } \\
\hline Acosmium bijugum (Vogel) Yakovlev & $\mathrm{Ab}$ & 33375 & arb & 1,66 \\
\hline Centrosema brasilianum (L.) Benth. & $\mathrm{Cb}$ & 33416 & herb & \\
\hline C. coriaceum Benth. & $\mathrm{Cc}$ & 33440 & herb & \\
\hline Moldenhawera nutans L.P. Queiroz, G.P. Lewis \& Allkin & Mn & 33432 & arb & \\
\hline Poecilanthe itapuana G.P. Lewis & Pit & 33384 & arb & \\
\hline Stilosanthes viscosa (L.) Sw. & $\mathrm{Sv}$ & 33398 & subarb & \\
\hline Swartzia apetala var. subcordata R.S. Cowan & As & 33391 & arb & 1,66 \\
\hline Vigna halophila (Piper) Maréchal, Mascherpa \& Stainier & $\mathrm{Vh}$ & 33439 & trep & \\
\hline \multicolumn{5}{|l|}{ HUMIRIACEAE } \\
\hline Humiria balsamifera var. parvifolia (A. Juss.) Cuatrec. & $\mathrm{Hb}$ & 33394 & arb & \\
\hline \multicolumn{5}{|l|}{ ICACINACEAE } \\
\hline Emmotum affine Miers & $\mathrm{Ea}$ & 33390 & arb & \\
\hline \multicolumn{5}{|l|}{ KRAMERIACEAE } \\
\hline Krameria bahiana B.B. Simpson & $\mathrm{Kb}$ & 33372 & subarb & \\
\hline \multicolumn{5}{|l|}{ LAMIACEAE } \\
\hline Eriope blanchetii (Benth.) R.M. Harley & $\mathrm{Eb}$ & 33366 & arb & 2,76 \\
\hline \multicolumn{5}{|l|}{ LAURACEAE } \\
\hline Ocotea notata (Nees \& Mart. ex Nees) Mez & On & 33419 & arb & \\
\hline \multicolumn{5}{|l|}{ LORANTHACEAE } \\
\hline Struthanthus polyrrhizus (Mart. ex Roem. \& Schult.) Mart. ex G. Don. & $\mathrm{Sp}$ & 33403 & herb-hemip & \\
\hline S. flexilis (Rusby) Kuijt & Sf & 33402 & herb-hemip & \\
\hline \multicolumn{5}{|l|}{ LYTHRACEAE } \\
\hline Cuphea brachiata (Mart.) Koehne & Cbr & 33360 & subarb & \\
\hline \multicolumn{5}{|l|}{ MALPIGHIACEAE } \\
\hline Byrsonima sericea DC. & Bs & 33445 & $\operatorname{arv}$ & \\
\hline B. coccolobifolia Kunth & $\mathrm{Bc}$ & 33369 & arv & 0,55 \\
\hline B. microphylla A. Juss. & $\mathrm{Bm}$ & 33358 & arb & 17,15 \\
\hline Stigmaphyllon paralias A. Juss. & $\mathrm{Sp}$ & 33408 & subarb & \\
\hline \multicolumn{5}{|l|}{ MELASTOMATACEAE } \\
\hline Comolia ovalifolia (DC.) Triana & Co & 33364 & subarb & \\
\hline Tibouchina bradeana Renner & $\mathrm{Tb}$ & 33376 & subarb & \\
\hline \multicolumn{5}{|l|}{ MIMOSACEAE } \\
\hline Abarema filamentosa (Benth.) Pittier & Af & 33361 & arb & \\
\hline \multicolumn{5}{|l|}{ MYRTACEAE } \\
\hline Calycolpus legrandii Mattos & $\mathrm{Cl}$ & 33359 & arb & 1,11 \\
\hline Eugenia pistaciifolia DC. & Epi & 33406 & arb & 1,11 \\
\hline Myrcia melanosepala Kiaersk. & $\mathrm{Mm}$ & 33409 & $\operatorname{arv}$ & \\
\hline Myrcia guianensis (Aubl.) DC. & $\mathrm{Mg}$ & 33396 & arb & 0,55 \\
\hline \multicolumn{5}{|l|}{ OCHNACEAE } \\
\hline Ouratea rotundifolia (Gardner) Engl. & Or & 33397 & arv & \\
\hline \multicolumn{5}{|l|}{ PASSIFLORACEAE } \\
\hline Passiflora edulis Sims & $\mathrm{Pe}$ & 33435 & trep & \\
\hline \multicolumn{5}{|l|}{ POLYGALACEAE } \\
\hline Polygala trichosperma Chod. \& Hub. & $\mathrm{Pt}$ & 33388 & subarb & \\
\hline Polygala violacea Aubl. & $\mathrm{Pv}$ & 33438 & subarb & \\
\hline
\end{tabular}


continuação

\begin{tabular}{|c|c|c|c|c|}
\hline Família/Espécie & Cód. & "Voucher" & Hábito & Ind ha ${ }^{-1}$ \\
\hline \multicolumn{5}{|l|}{ POLYGONACEAE } \\
\hline Coccoloba cordifolia Meisn. & Cco & 33365 & arb & 1,11 \\
\hline C. ramosissima Wedd. & Cra & 33412 & arb & 1,11 \\
\hline \multicolumn{5}{|l|}{ RUBIACEAE } \\
\hline Mitracarpus frigidus var. discolor (Miq.) K. Schum & Mf & 33426 & subarb & \\
\hline Borreria cymosa (Spreng.) Cham. \& Schltdl. & $\mathrm{Bc}$ & 33436 & herb & \\
\hline B. verticillata (L.) G. Mey. & $\mathrm{Bv}$ & 33427 & subarb & \\
\hline Guettarda platypoda DC. & Gp & 33386 & arb & 0,55 \\
\hline Rudgea crassifolia Zappi \& E. Lucas & $\mathrm{Rc}$ & 33422 & arb & \\
\hline \multicolumn{5}{|l|}{ SAPINDACEAE } \\
\hline Cardiospermum integerrimum Radlk. & $\mathrm{Ci}$ & 33404 & li & \\
\hline \multicolumn{5}{|l|}{ SAPOTACEAE } \\
\hline Manilkara salzmannii (DC.) H.J. Lam. & Ms & 33405 & arb & 2,21 \\
\hline \multicolumn{5}{|l|}{ SOLANACEAE } \\
\hline Solanum stagnale Moric. & Ss & 33380 & arb & 0,55 \\
\hline \multicolumn{5}{|l|}{ STERCULIACEAE } \\
\hline Waltheria cinerascens St. Hilaire & Wc & 33377 & arb & 23,23 \\
\hline \multicolumn{5}{|l|}{ THEACEAE } \\
\hline Ternstroemia sp. & Tsp & 33444 & arb & 0,55 \\
\hline \multicolumn{5}{|l|}{ VELLOZIACEAE } \\
\hline Vellozia dasypus Seub. & $\mathrm{Vd}$ & 33373 & herb & \\
\hline \multicolumn{5}{|l|}{ VERBENACEAE } \\
\hline Lantana camara L. & $\mathrm{Lc}$ & 33411 & subarb & \\
\hline Vitex cymosa Bertero ex Spreng. & $\mathrm{Vc}$ & 33392 & arb & 5,53 \\
\hline
\end{tabular}

Tabela 2. Índices de Sørensen, expresso em porcentagem, para a similaridade florística (espécies e gêneros) entre as comunidades de plantas apícolas das dunas de Abaeté, Bahia, e de ecossistemas semelhantes nos Estados do Maranhão (MA), Paraíba (PB), Paraná (PR) e Rio Grande do Sul (RS).

Table 2. Sørensen indexes, in percentage, of floristic similarities (species and genera) among bee plant communities in the sea coastal sand dunes of Abaeté, Bahia and those of similar ecosystems in states of Maranhão (MA), Paraíba (PB), Paraná (PR) e Rio Grande do Sul (RS).

\begin{tabular}{|c|c|c|}
\hline Estados & $\mathrm{BA}\left(12^{\circ} 56^{\prime} \mathrm{S}\right)$ & Fontes \\
\hline \multicolumn{3}{|c|}{ Espécies } \\
\hline MA $\left(2^{\circ} 30^{\prime} \mathrm{S}\right.$ & 6,1 & Albuquerque (1998) \\
\hline $\mathrm{PB}\left(7^{\circ} 3^{\prime} \mathrm{S}\right)$ & 10,5 & Madeira-da-Silva (1998) \\
\hline $\operatorname{PR}\left(25^{\circ} 30^{\prime} \mathrm{S}\right)$ & 3,9 & $\begin{array}{l}\text { Zanela (1991), Schwartz Filho } \\
\text { (1993), Laroca (1974) }\end{array}$ \\
\hline $\mathrm{RS}\left(30^{\circ} \mathrm{S}\right)$ & 0 & Alves-dos-Santos (1999) \\
\hline \multicolumn{3}{|c|}{ Gêneros } \\
\hline MA $\left(2^{\circ} 30^{\prime} \mathrm{S}\right)$ & 18,2 & Albuquerque (1998) \\
\hline $\mathrm{PB}\left(7^{\circ} 3^{\prime} \mathrm{S}\right)$ & 25,3 & Madeira-da-Silva (1998) \\
\hline $\mathrm{PR}\left(25^{\circ} 30^{\prime} \mathrm{S}\right)$ & 11,7 & $\begin{array}{l}\text { Zanela (1991), Schwartz Filho } \\
\text { (1993), Laroca (1974) }\end{array}$ \\
\hline $\mathrm{RS}\left(30^{\circ} \mathrm{S}\right)$ & 9,4 & Alves-dos-Santos (1999) \\
\hline
\end{tabular}

Em Abaeté, mais de 50\% das espécies floresceram por período superior a seis meses, e o pico de floração para a maioria delas ocorreu entre setembro e dezembro (tabela 3). Poucos taxa, como Tabebuia, Vellozia, Coccoloba, Agarista e Tibouchina, apresentaram acentuada sazonalidade. $\mathrm{O}$ número de indivíduos floridos por espécie variou de 1 a mais de 400 (figura 1A). O pico de indivíduos floridos, em agosto, deveu-se ao elevado número de indivíduos de W. cinerascens, C. ramosa, B. cymosa e Polygala trichosperma.

O maior número de flores no campo ocorreu de janeiro a março e de novembro a dezembro (figura 1B), coincidindo com o período de menor precipitação (figura 2). Muitas espécies apresentaram grande abundância de flores reunidas em densas inflorescências, com o número médio de flores por indivíduo variando de 10.000 em Tapirira guianensis e Coccoloba cordifolia, a mais de $50.000 \mathrm{em}$ Humiria balsamifera, Byrsonima sericea, Acosmium bijugum, Protium icicariba e P. bahianum.

Doze espécies foram predominantemente visitadas por abelhas, em Abaeté, havendo variação diária e sazonal na abundância relativa de visitantes (figuras 3 e 4). $C$. brachiata recebeu o maior número de visitas 
Tabela 3. Período de floração e abelhas visitantes (número de espécies e indivíduos) coletados nas flores, entre janeiro e dezembro de 1996, nas dunas de Abaeté, Salvador, Bahia. As espécies foram ordenadas conforme a duração da floração

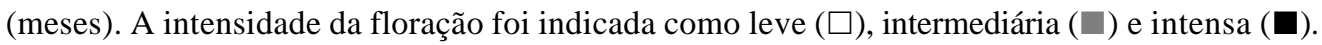

Table 3. Blooming period and visitors bee (number of species and of individuals) collected on flowers between January and December, 1996, in the sea coastal sand dunes of Abaeté, Salvador, Bahia. Species were ordered according to flowering period length (in months). Flowering intensity was indicated as light $(\square)$, intermediate ( $\square$ ) and intensive (ם).

\begin{tabular}{llllllllllllllll}
\hline Espécie vegetal & J & F & M & A & M & J & J & A & S & O & N & D \\
$(>5$ & meses $)$
\end{tabular}

Visitantes

Indivíduos Espécies

Byrsonima microphylla

Cuphea brachiata

Chamaecrista ramosa

Krameria bahiana

Lepidaploa arenaria

Waltheria cinerascens

Humiria balsamifera

Dactylaena microphylla

Eriope blanchetii

Lantana camara

Stigmaphyllon paralias

Comolia ovalifolia

Cardiospermum integerrimum

Vitex cymosa

Tapirira guianensis

Stilosanthes viscosa

Solanum stagnale

Evolvulus maximiliani

Guettarda platypoda

Struthanthus polyrrhizos

Calycolpus legrandii

Kielmeyera argentea

Myrcia guianensis

Mandevilla funiformis

Emmotum affine

Manilkara salzmannii

Mikania nitida

Conocliniopsis prasiifolia

Coccoloba cordifolia

Tibouchina bradeana

Rudgea crassifolia

Euphorbia gymnoclada

Ditassa blanchetii

Allagoptera brevicalyx

Coccoloba ramosissima

Protium icicariba

Polygala trichosperma

Myrcia melanosepala

Borreria verticillata

Centrosema coriaceum

C. brasilianum

Lepidaploa edmundoi

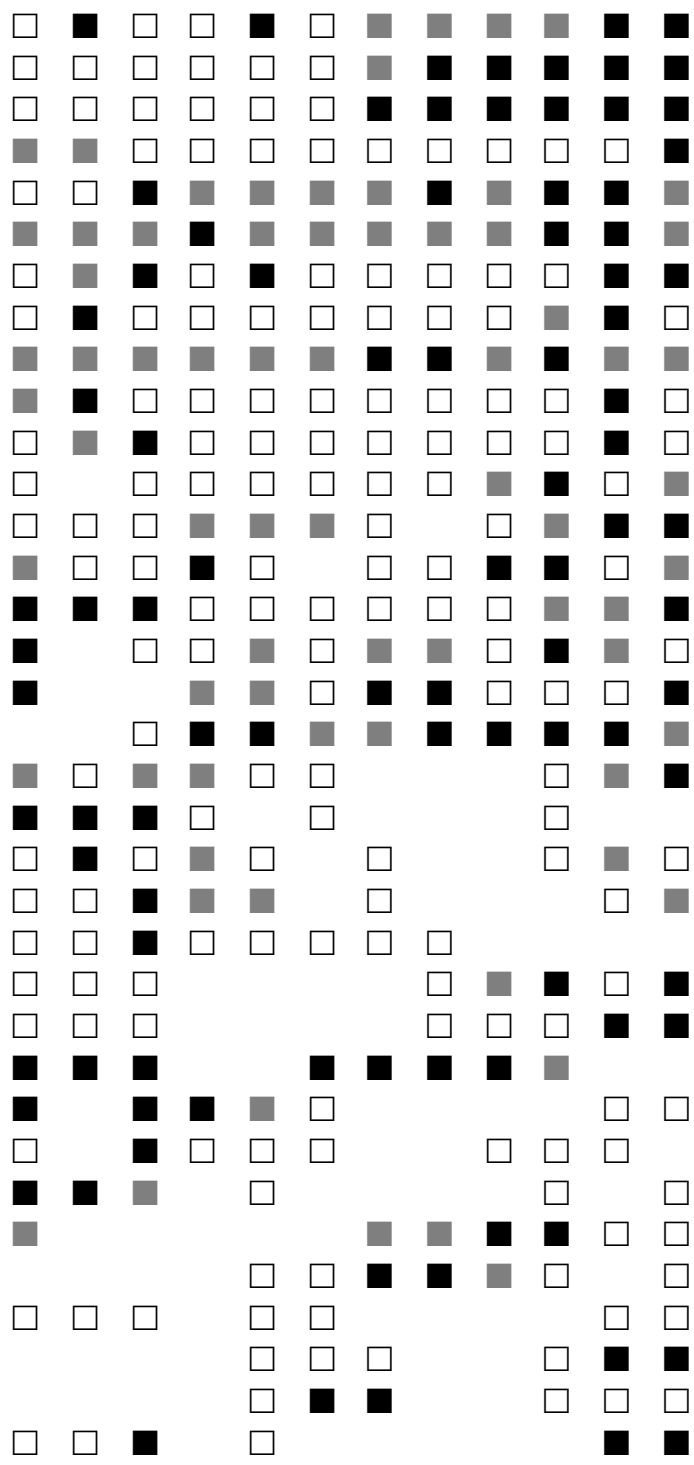

(2 a 5 meses)

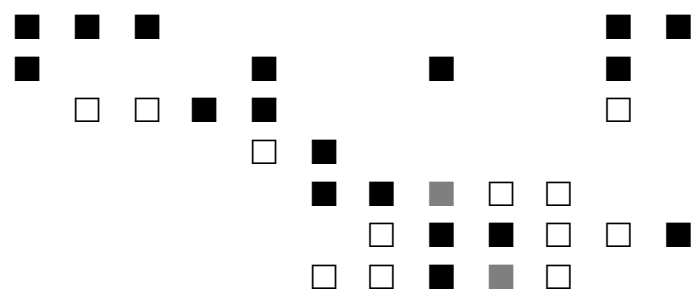

$\begin{array}{rr}143 & 12 \\ 646 & 22 \\ 158 & 15 \\ 47 & 7 \\ 61 & 7 \\ 580 & 16 \\ 364 & 9 \\ 11 & 4 \\ 315 & 21 \\ 5 & 1 \\ 1 & 1 \\ 15 & 3 \\ 14 & 4 \\ 28 & 4 \\ 159 & 6 \\ 20 & 5 \\ 14 & 3 \\ 5 & 4 \\ 8 & 1 \\ 79 & 6 \\ 9 & 6 \\ 19 & 5 \\ 35 & 3 \\ 1 & 1 \\ 7 & 3 \\ 165 & 3 \\ 34 & 8 \\ 13 & 6 \\ 125 & 2 \\ 13 & 5 \\ 20 & 2 \\ 1 & 1 \\ 1 & 1 \\ 3 & 1 \\ 51 & 1\end{array}$

$\begin{array}{rl}65 & 4 \\ 7 & 4 \\ 6 & 1 \\ 10 & 4 \\ 3 & 2 \\ 17 & 7 \\ 3 & 2\end{array}$

continua 
continuação

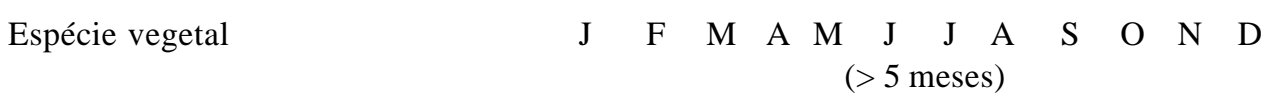

Visitantes

Indivíduos Espécies

Struthanthus flexilis

Eugenia pistaciifolia

Acosmium bijugum

Tabebuia elliptica

Moldenhawera nutans

Byrsonima coccolobifolia

Vellozia dasypus

Anthurium longipes

Ouratea rotundifolia

Abarema filamentosa

Mitracarpus frigidus

B. cymosa

Swartzia apetala

Agarista revoluta

Protium bahianum

Ocotea notata

Passiflora edulis

Stilpnopappus scaposus

Ternstroemia sp.

Byrsonima sericea

Aechmea sp.
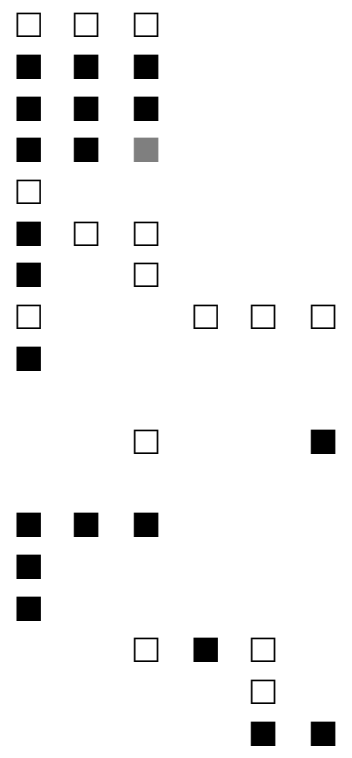

(1 mês)

Poecilanthe itapuana

Polygala sp.

Total de visitantes

$\begin{array}{rr}3 & 2 \\ 1 & 1 \\ 342 & 22 \\ 32 & 7 \\ 4 & 4 \\ 2 & 1 \\ 4 & 1 \\ 4 & 1 \\ 8 & 3 \\ 3 & 1 \\ 16 & 6 \\ 87 & 4 \\ 1 & 1 \\ 11 & 3 \\ 33 & 5 \\ 8 & 1 \\ 10 & 5 \\ 1 & 1 \\ 1 & 1 \\ 57 & 8 \\ 1 & 1\end{array}$

$\begin{array}{rrr}1 & 1 & \\ 1 & & 1 \\ 983 & & 49\end{array}$

de Apoidea (646). W. cinerascens e C. brachiata, floresceram ao longo de todo o ano (tabela 3 ). $\mathrm{Na}$ maioria das espécies, as flores permaneceram abertas durante todo o dia e, às vezes, por mais de um dia. Entre as espécies predominantemente visitadas por Apoidea, Chamaecrista ramosa e Struthanthus polyrrhizus, cujas flores murcharam no período da tarde, representaram exceção.

As plantas com flores com características melitófilas representaram $78 \%$ do total observado. A maioria era pequena, tubular, pouco conspícua e formando inflorescências compactas (tabela 4). As cores observadas foram lilás $(32 \%)$, creme $(31 \%)$, branca $(17 \%)$, amarelas $(15 \%)$, rosa $(3 \%)$ e alaranjada $(2 \%)$. A actinomorfia $(63 \%)$ predominou em relação a zigomorfia $(37 \%)$. O número de flores monoclinas $(89 \%)$ superou o de flores diclinas $(11 \%)$.

O principal recurso produzido pelas flores foi o néctar $(66 \%)$, seguido de pólen $(26 \%)$ e óleos $(8 \%)$. Nas espécies de Melastomataceae, Solanaceae,
Caesalpiniaceae, Mimosaceae e Ochnaceae, o pólen foi o único recurso disponível. Apenas Malpighiaceae e Krameriaceae apresentaram óleos como recursos primários, sendo que nestas o pólen foi também coletado pelas abelhas.

\section{Discussão}

A flora fanerogâmica da APA das Dunas e Lagoas do Abaeté é mais rica (Britto et al. 1993) do que a de outras áreas de dunas no Brasil (Araújo \& Henriques 1984). Em relação às famílias mais importantes, em número de espécies, excetuando-se o Rio Grande do Sul, as demais áreas assemelharam-se bastante. Fabaceae foi a família com maior riqueza específica na Bahia, Paraíba e Maranhão e a segunda no Paraná. Estudos fitossociológicos realizados em diversas áreas de restinga, ao longo da costa brasileira (Araújo \& Henriques 1984, Pinto et al. 1984, Furlan et al. 1990, Pereira \& Gomes 1994, Silva et al. 1994), destacaram 


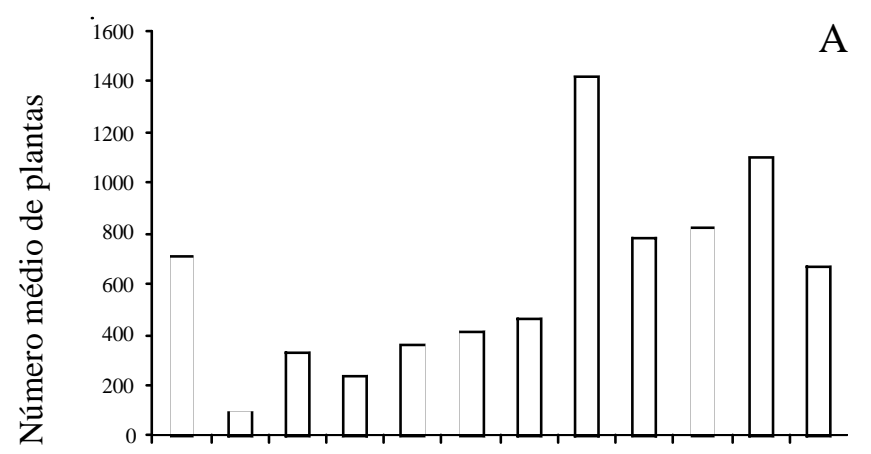

$\begin{array}{llllllllllll}\text { J } & \text { F } & \text { M } & \text { A } & \text { M } & \text { J } & \text { J } & \text { A } & \text { S } & \text { O } & \text { N } & \text { D }\end{array}$

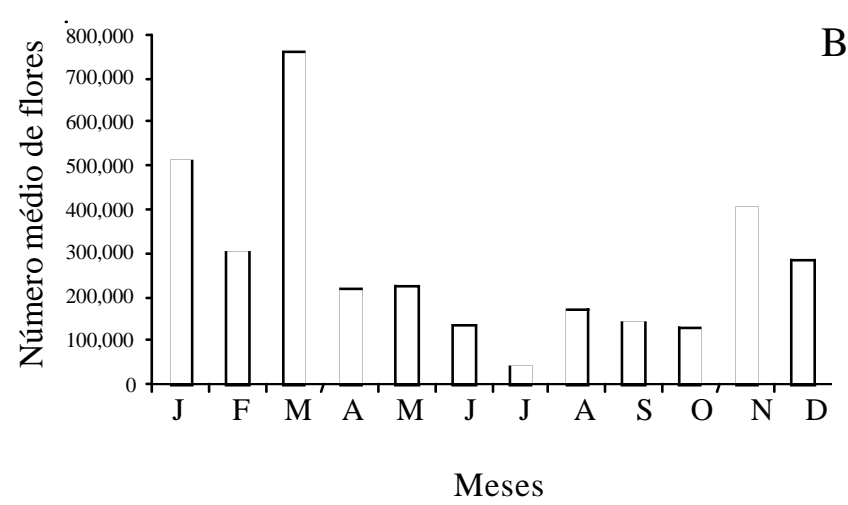

Figura 1. Número de indivíduos floridos (A) e a produção de flores nas espécies vegetais visitadas por abelhas (B) no ano de 1996, nas dunas de Abaeté, Salvador, Bahia.

Figure 1. Number of flowering individuals (A) and flower production in plant species visited by bees (B) in 1996, in the sea coastal sand dunes of Abaeté, Salvador, Bahia.

Leguminosae (incluindo Fabaceae, Caesalpiniaceae e Mimosaceae), juntamente com Rubiaceae, Myrtaceae, Asteraceae, Bromeliaceae, Orchidaceae e Euphorbiaceae, como as famílias mais representativas em espécies nesse ecossistema. Segundo Polhill et al. (1981), a representatividade de Leguminosae nas planícies litorâneas deve-se à diversidade de hábitos, preferências ecológicas e mecanismos de manutenção, defesa e reprodução, por ela apresentados.

$\mathrm{Na}$ área de estudo, a maior proporção de espécies com hábito arbustivo está associada ao fato de as dunas de Abaeté serem internas, relativamente estáveis, e pela menor salinidade do substrato, devido à elevação do lençol freático em direção às dunas interiores, quando comparado com outras áreas de dunas costeiras no país (Morawetz 1983).

Dentre as plantas visitadas por abelhas, algumas apresentam ampla distribuição geográfica, ocorrendo em outras áreas de restinga, ou mesmo no interior do Brasil, como Humiria balsamifera, Eugenia pistaciifolia, Stylosanthes viscosa e Allagoptera brevicalyx. Outras espécies são endêmicas de Abaeté, como Aechmea itapoana (Morawetz \& Till 1981), Moldenhawera abaetana (L.P. Queiroz \& R. Allkin, dados não publicados) e Poecilante itapoana (Lewis 1989). Há também algumas espécies ruderais e invasoras, como Conocliniopsis prasiifolia, Centrosema coriaceum, Passiflora edulis e Lantana camara, o que evidencia a interferência antrópica na área de estudos, embora ainda pequena.

A flora apícola de Abaeté apresenta maior riqueza que os ecossistemas similares do nordeste brasileiro. Albuquerque (1998) identificou, em uma área restrita de dunas em São Luís, MA, 33 espécies visitadas por abelhas. Silva \& Martins (1999), em uma área de restinga próxima a João Pessoa, PB, encontraram 29 espécies visitadas. Segundo Morawetz (1983), muitos taxa são substituídos por vicariantes cogenéricos ao longo da costa brasileira, como é o caso dos gêneros Aechmea, Coccoloba, Davilla, Guettarda, Protium, Vitex e Swartzia, o que explicaria a similaridade observada em nível genérico.

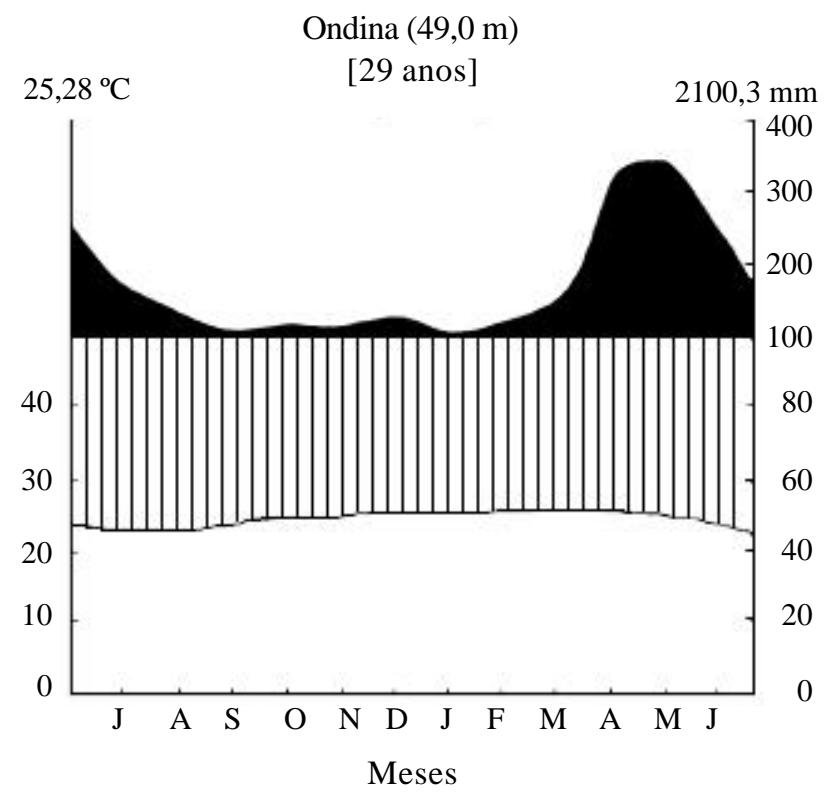

Figura 2. Diagrama ombrotérmico de Salvador, segundo Walter (1963), no período entre 1961-1990. (Fonte: Departamento Nacional de Meteorologia - DNMET, estação meteorológica de Ondina, Salvador). $(\mathbf{\square}=$ Período superúmido; 血 = Período úmido).

Figure 2. Ombrothermic diagram from Salvador according to Walter (1963) between 1961-1990. (Source: Departamento Nacional de Meteorologia - DNMET, Ondina's meteorologic station, Salvador). ( $\square=$ Super wet season; [Il = West season). 


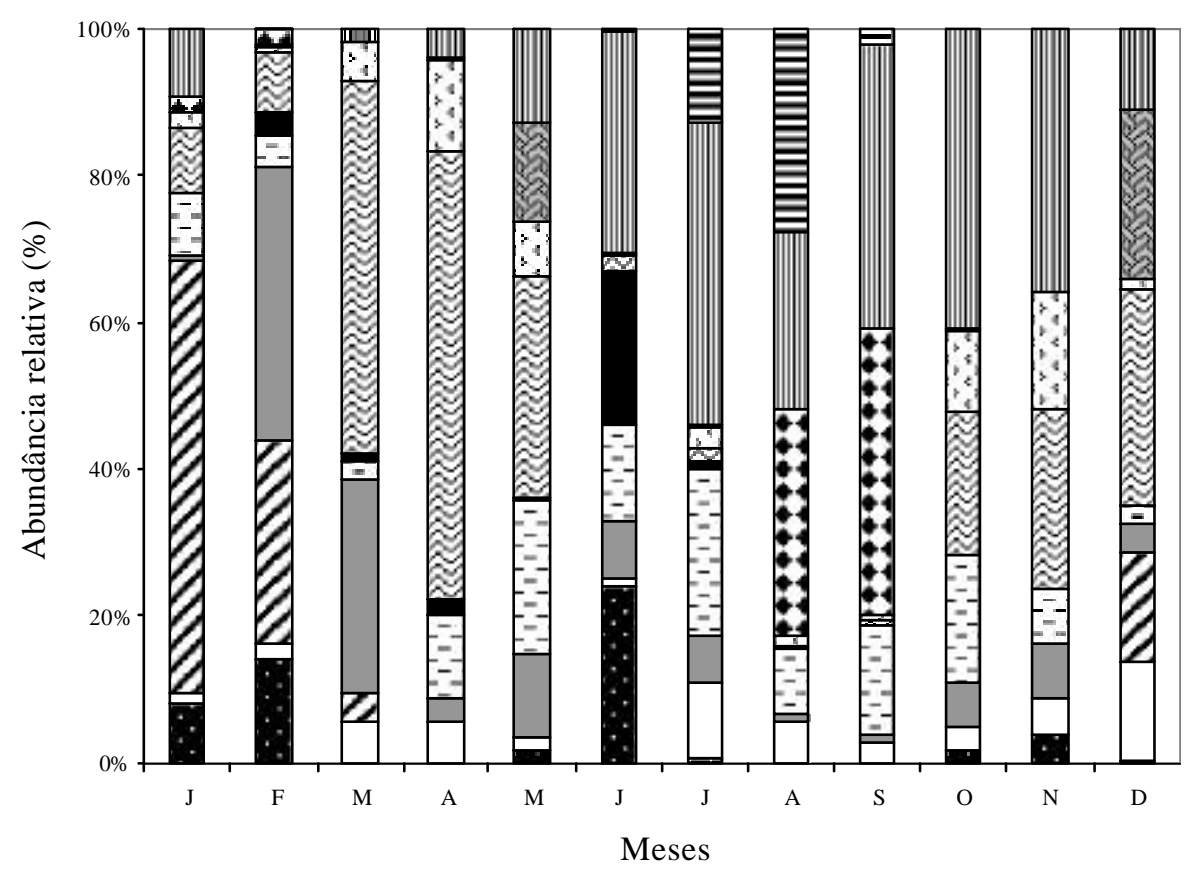

Figura 3. Abundância relativa (\%) mensal de visitas das abelhas às espécies vegetais predominantemente visitadas em Abaeté, Salvador, Bahia, no ano de 1996.

Figure 3. Monthly relative abundance $(\%)$ of bee visits to the predominantly bee visited species in Abaeté, Salvador, Bahia, in the year 1996.

( $\mathbf{0}=$ Tapirira guianensis; 国=Acosmium bijugum; $\square=$ Eriope blanchetii; $\square=$ Cuphea brachiata; $\mathbf{\square}=$ Coccolobacordifolia $;$ $\square=$ Walteheria cinerascens; $\square=$ Chamaecrista ramosa var. ramosa $; \square=$ Humiria balsamifera var. parvifolia; $\square=$ Struthanthus polyrrhizus; $\square=$ Byrsonima microphylla $; \square=$ Manilkara salzmannii; $\square=$ Borreria cymosa).

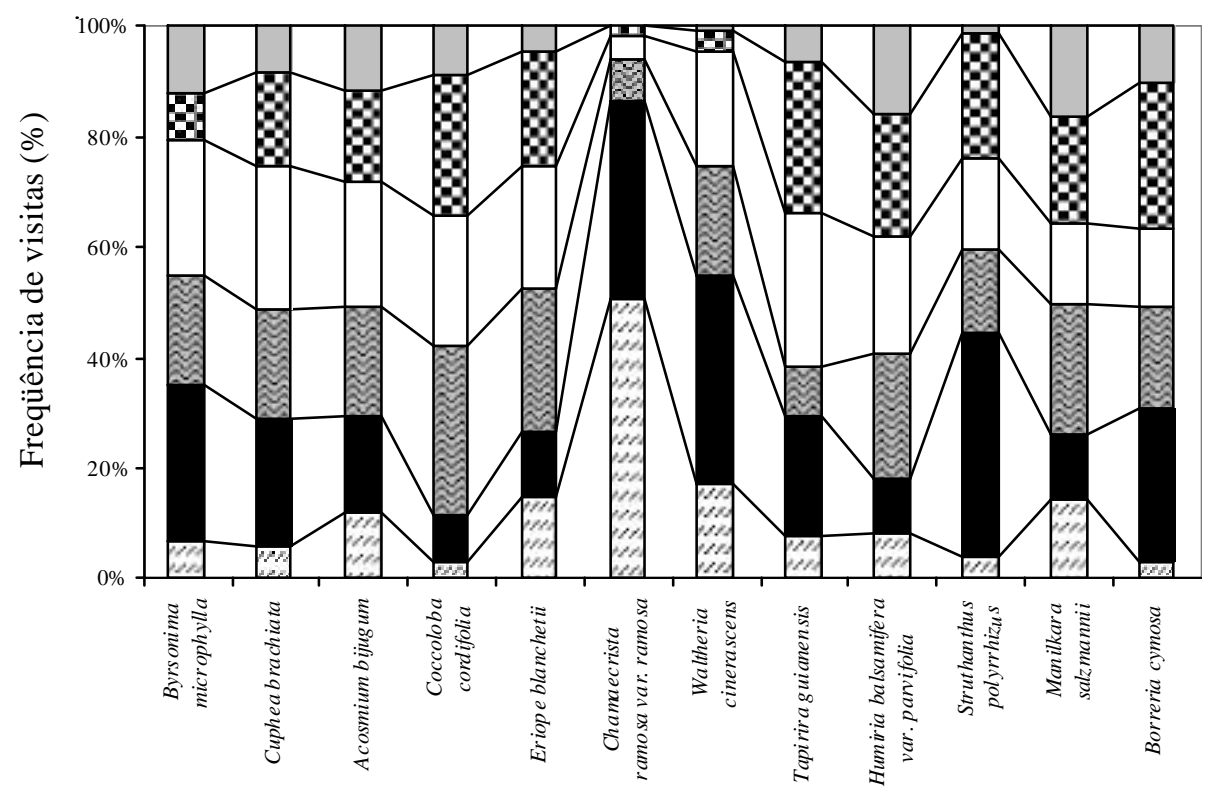

Espécies vegetais

Figura 4. Freqüência (\%) de visitas das abelhas às plantas predominantemente visitadas nas diferentes horas do dia em Abaeté, Salvador, Bahia.

Figure 4. Frequency (\%) of visits by Apoidea to the predominantly bee visited plants along day time in Abaeté, Salvador, Bahia. ( $\square=6: 00-8: 00 ; \square=8: 00-10: 00 ; \square=10: 00-12: 00 ; \square=12: 00-14: 00 ; \square=14: 00-16: 00 ; \square=16: 00-18: 00$ ). 
Tabela 4. Principais características florais das espécies vegetais visitadas por abelhas nas dunas de Abaeté, Salvador, Bahia: cor $(\mathrm{a}=$ alva; $\mathrm{am}=$ amarela; $\mathrm{c}=$ creme; li = lilás; $\mathrm{r}=$ rosa $)$, diâmetro e profundidade da corola $(\mathrm{em} \mathrm{cm})$, simetria $($ act $=$ actinomorfa e zig =zigomorfa $)$, recurso coletado pelo visitante, deiscência da antera $(\mathrm{L}=$ longitudinal; $\mathrm{P}=$ poricida; $\mathrm{V}=$ valvar $)$ e sexualidade.

Table 4. Main floral characteristics of plant species visited by bees in the sea coastal sand dunes of Abaeté, Salvador, Bahia: color $(\mathrm{a}=$ white; $\mathrm{am}=$ yellow; $\mathrm{c}=$ cream; $\mathrm{li}=$ lilac; $\mathrm{r}=$ pink), diameter and depth of corolla $(\mathrm{in} \mathrm{cm})$, symmetry (act = actinomorphous and zig $=$ zigomorphous $)$, resource collected by visitor, anther dehiscence $(\mathrm{L}=$ longitudinal; $\mathrm{P}=$ poricidal; $\mathrm{V}=\mathrm{valvate})$ and sexuality.

\begin{tabular}{|c|c|c|c|c|c|c|c|}
\hline \multirow[b]{2}{*}{ Espécie } & \multicolumn{3}{|c|}{ Características da corola } & \multirow[b]{2}{*}{ Simetria } & \multirow{2}{*}{$\begin{array}{l}\text { Recurso } \\
\text { coletado }\end{array}$} & \multirow{2}{*}{$\begin{array}{l}\text { Deiscência } \\
\text { da antera }\end{array}$} & \multirow{2}{*}{ Sexualidade } \\
\hline & Cor & Diâmetro & Profundidade & & & & \\
\hline$B m$ & $\mathrm{r}$ & 1,42 & 0 & zig & óleo & $\mathrm{L}$ & monoclina \\
\hline$C l$ & $\mathrm{a}$ & 4,50 & 0 & act & pólen & $\mathrm{L}$ & monoclina \\
\hline $\mathrm{Cbr}$ & am & 1,36 & 0,96 & zig & néctar & $\mathrm{L}$ & monoclina \\
\hline$A b$ & $\mathrm{c}$ & 1,20 & 0 & act & néctar & $\mathrm{L}$ & monoclina \\
\hline$M n$ & $\mathrm{c}$ & 0,52 & 0,66 & act & néctar & $\mathrm{L}$ & monoclina \\
\hline$K a$ & $\mathrm{a}$ & 6,00 & 0 & act & pólen & $\mathrm{L}$ & monoclina \\
\hline Co & li & 3,05 & 0 & act & pólen & $\mathrm{P}$ & monoclina \\
\hline$C c$ & $\mathrm{a}$ & 0,36 & 0,38 & act & pólen & $\mathrm{L}$ & diclina \\
\hline$E b$ & li & 1,10 & 0,46 & zig & néctar & $\mathrm{L}$ & monoclina \\
\hline$M n$ & $\mathrm{a}$ & 3,28 & 0 & zig & pólen & $\mathrm{P}$ & monoclina \\
\hline$B c$ & $\mathrm{a}$ & 1,72 & 0 & zig & óleo & $\mathrm{L}$ & monoclina \\
\hline $\mathrm{Cr}$ & am & 2,10 & 0 & zig & pólen & $\mathrm{P}$ & monoclina \\
\hline$K b$ & li & 2,10 & 0 & zig & óleo/pólen & $\mathrm{P}$ & monoclina \\
\hline$V d$ & $\mathrm{li}$ & 7,70 & 1,20 & act & pólen & $\mathrm{L}$ & monoclina \\
\hline$M f$ & am & 9,28 & 3,44 & act & néctar & $\mathrm{L}$ & monoclina \\
\hline$E g$ & $\mathrm{a}$ & $*$ & $*$ & $*$ & $*$ & $*$ & * \\
\hline$T b$ & li & 3,82 & 0 & act & pólen & $\mathrm{P}$ & monoclina \\
\hline$W c$ & am & 0,86 & 0,68 & act & néctar & $\mathrm{L}$ & monoclina \\
\hline$E b$ & li & 0,42 & 0,40 & act & néctar & $\mathrm{L}$ & monoclina \\
\hline Ss & li & 5,3 & 0 & act & pólen & $\mathrm{P}$ & monoclina \\
\hline$V a$ & li & 0,56 & 0,98 & act & néctar & $\mathrm{L}$ & monoclina \\
\hline$T g$ & $\mathrm{a}$ & 0,44 & 0 & act & néctar & $\mathrm{L}$ & monoclina \\
\hline$P i$ & $*$ & $*$ & $*$ & zig & * & $\mathrm{L}$ & monoclina \\
\hline Pic & $\mathrm{c}$ & 0,25 & 0,28 & act & néctar & $\mathrm{L}$ & diclina \\
\hline$G p$ & $\mathrm{a}$ & 0,95 & 1,10 & act & $*$ & $\mathrm{~L}$ & monoclina \\
\hline$P p$ & $\mathrm{c}$ & 0,20 & 0,40 & zig & $*$ & $\mathrm{P}$ & monoclina \\
\hline$A r$ & $\mathrm{c}$ & 0,20 & 0,86 & act & néctar/pólen & $\mathrm{P}$ & monoclina \\
\hline$E a$ & $\mathrm{c}$ & 0,84 & 0,22 & act & pólen & $\mathrm{L}$ & monoclina \\
\hline $\mathrm{Sa}$ & & 0,76 & 0 & zig & pólen & $\mathrm{L}$ & monoclina \\
\hline$V c$ & li & 1,46 & 0,70 & zig & néctar & $\mathrm{L}$ & monoclina \\
\hline$H b$ & $\mathrm{c}$ & 0,78 & 0 & act & néctar & $*$ & monoclina \\
\hline$M m$ & $\mathrm{a}$ & 0,96 & 0 & act & pólen? & $\mathrm{L}$ & monoclina \\
\hline$O r$ & $\mathrm{c}$ & 0,5 & 1,5 & $*$ & $*$ & $*$ & $*$ \\
\hline$S v$ & am & 0,52 & 0,84 & zig & néctar? & $\mathrm{L}$ & monoclina \\
\hline$D m$ & li & 0,26 & 0,40 & act & néctar & $\mathrm{L}$ & monoclina \\
\hline$T e$ & li & 5,16 & 3,50 & zig & néctar & $\mathrm{L}$ & monoclina \\
\hline$P b$ & $\mathrm{c}$ & 0,15 & 0,33 & act & néctar & $\mathrm{L}$ & diclina \\
\hline$S f$ & $\mathrm{c}$ & 0,66 & 0,30 & act & néctar & $\mathrm{L}$ & monoclina \\
\hline$S p$ & $\mathrm{c}$ & 0,70 & 0,20 & act & néctar & $\mathrm{L}$ & monoclina \\
\hline$C i$ & $*$ & 0,43 & 0 & zig & néctar & $\mathrm{L}$ & diclina \\
\hline$M s$ & $\mathrm{c}$ & 0,72 & 0,25 & act & néctar & $\mathrm{L}$ & monoclina \\
\hline Epi & $\mathrm{a}$ & 0,80 & 0 & act & $*$ & $\mathrm{~L}$ & monoclina \\
\hline $\mathrm{Sp}$ & am & 2,87 & 0 & zig & óleo & $\mathrm{L}$ & monoclina \\
\hline
\end{tabular}


continuação

\begin{tabular}{|c|c|c|c|c|c|c|c|}
\hline \multirow[b]{2}{*}{ Espécie } & \multicolumn{3}{|c|}{ Características da corola } & \multirow[b]{2}{*}{ Simetria } & \multirow{2}{*}{$\begin{array}{l}\text { Recurso } \\
\text { coletado }\end{array}$} & \multirow{2}{*}{$\begin{array}{c}\text { Deiscência } \\
\text { da antera }\end{array}$} & \multirow{2}{*}{ Sexualidade } \\
\hline & Cor & Diâmetro & Profundidade & & & & \\
\hline$M g$ & $\mathrm{a}$ & 0,80 & 0 & act & pólen? & $\mathrm{L}$ & monoclina \\
\hline$L c$ & li & 0,72 & 0,46 & zig & néctar & $\mathrm{L}$ & monoclina \\
\hline $\mathrm{Cra}$ & $\mathrm{c}$ & 0,25 & 0,10 & zig & néctar & $*$ & monoclina \\
\hline$A f$ & $\mathrm{c}$ & 7,00 & 0 & act & néctar & $*$ & monoclina \\
\hline Em & $\mathrm{li}$ & 0,97 & 0,10 & act & néctar & $\mathrm{L}$ & monoclina \\
\hline On & $*$ & 0,20 & 0 & act & $*$ & $\mathrm{~V}$ & diclina \\
\hline$R c$ & $\mathrm{a}$ & 0,50 & 1,20 & act & néctar & $\mathrm{L}$ & monoclina \\
\hline$D b$ & $\mathrm{c}$ & 0,46 & 0 & act & néctar & $\mathrm{L}$ & monoclina \\
\hline$M f$ & $*$ & 0,16 & 0,36 & act & $*$ & $\mathrm{~L}$ & monoclina \\
\hline$B v$ & $*$ & 0,20 & 0,1 & act & $*$ & $\mathrm{~L}$ & monoclina \\
\hline Ss & $\mathrm{li}$ & 0,15 & 0,38 & act & néctar & $\mathrm{L}$ & monoclina \\
\hline$A b r$ & $\mathrm{c}$ & 0,80 & 0,30 & $*$ & $*$ & $*$ & $*$ \\
\hline$C c$ & li & 4,80 & 1,10 & zig & néctar & $\mathrm{L}$ & monoclina \\
\hline$P e$ & li & 8,00 & 0 & $*$ & $*$ & $*$ & $*$ \\
\hline$B c$ & $*$ & 0,30 & 0,44 & act & $*$ & $\mathrm{~L}$ & monoclina \\
\hline$L e$ & $\mathrm{li}$ & 0,10 & 0,64 & act & néctar & $\mathrm{L}$ & monoclina \\
\hline$P v$ & $\mathrm{c}$ & 0,42 & 0,44 & zig & $*$ & $\mathrm{P}$ & monoclina \\
\hline$V h$ & $\mathrm{li}$ & 1,46 & 0,88 & zig & néctar & $\mathrm{L}$ & monoclina \\
\hline$C b$ & li & 4,20 & 0,70 & zig & néctar & $\mathrm{L}$ & monoclina \\
\hline$T s p$ & $\mathrm{r}$ & $*$ & $*$ & zig & $*$ & $\mathrm{~L}$ & monoclina \\
\hline$B s$ & am & 1,14 & 0 & zig & óleo & $\mathrm{L}$ & monoclina \\
\hline
\end{tabular}

(*) indica informações não levantadas (indicates non-surveyed information); as abreviações das espécies correspondem àquelas utilizadas na tabela 1 (For species abbreviations see table 1).

O longo período de florescimento das espécies vegetais em Abaeté assemelha-se ao observado em ecossistemas similares na região nordeste (Albuquerque 1998, Silva \& Martins 1999) e nos campos rupestres (Faria 1994). Difere, no entanto, de outros ecossistemas tropicais, onde a maioria das espécies floresce por períodos curtos (Heithaus 1979, Pedro 1992, Martins 1995). Ao estudar a flora de Abaeté, Morawetz (1983) também observou pequena sazonalidade na área. Faria (1994) apontou que, em comunidades submetidas ao estresse (interferências antrópicas ou situações climáticas adversas), onde usualmente o sistema de polinização é generalista, predominam longos períodos de florescimento. Essa estratégia floral reduz a possibilidade de geitonogamia e força os polinizadores a procurar recursos nos coespecíficos (Bawa 1983).

Em Abaeté, como nas dunas do Maranhão (Albuquerque 1998), onde os ventos fortes e a elevada intensidade luminosa limitam a atividade dos visitantes, longos períodos de floração constituem vantagem adaptativa. Com relação à disponibilidade diária de flores, as espécies em Abaeté divergem daquelas das dunas do Maranhão (Albuquerque 1998), onde as plantas abrem entre $5 \mathrm{~h} 00$ e $6 \mathrm{~h} 00$, permanecendo abertas por pouco tempo.

A disponibilidade contínua de polinizadores, em sua maioria abelhas solitárias de vida longa do gênero Xylocopa (Viana et al. 2002), é um importante fator que permite a existência de variação temporal nos picos de floração entre as espécies. Nas espécies que utilizaram o mesmo grupo de vetores de pólen, como Walteria cinerascens e Cuphea brachiata, isso pode ser evidenciado pelas acentuadas diferenças nas abundâncias relativas dos visitantes, em certos meses do ano.

Especializações para polinização por abelhas ocorreram em elevada proporção de espécies vegetais ( $78 \%$ dentre as $85 \%$ visitadas por estes insetos) destacando-se, dentre elas, as espécies das famílias Caesalpiniaceae, Solanaceae e Melastomataceae, com anteras poricidas; e as espécies de Malpighiaceae e Krameriaceae, que possuem especializações para a secreção de óleos (os elaióforos), sugerindo, assim, que as abelhas devam ser os principais agentes polinizadores. Estudos de caso realizados com as espécies Eriope blanchetii (Silva et al. 2005) e Cuphea brachiata, cujas flores são especializadas, confirmaram 
a relevância das abelhas como polinizadores.

Dentre as plantas visitadas, predominam as espécies com flores pequenas, pouco conspícuas, monoclinas, com simetrias radiais e agrupadas em inflorescências. Essas inflorescências, mais conspícuas que as flores, devem representar a unidade funcional efetiva para atração dos polinizadores em Abaeté, como sugerido por Kevan et al. (1996).

De modo geral, o sistema abelha-flor nesse ambiente não se enquadra perfeitamente nas características previstas pelas síndromes florais descritas por Faegri \& Van der Pij1 (1979). A comunidade descrita ilustra bem o postulado por Proctor et al. (1996), de que as abelhas não podem ser associadas a uma única síndrome floral, mas sim a várias características florais, que envolvem, dentre outras, os modos de atração, como odor, cor, tipo e forma da flor e tipo de recurso oferecido aos visitantes. Vale ressaltar ainda, que espécies vegetais com adaptações associadas a outros polinizadores, como por exemplo, Agarista revoluta, associada a Lepidoptera, também constituem recursos alternativos para as abelhas nessas dunas, demonstrando assim, que as interações abelha-flor nesse ambiente podem ser bastante plásticas.

Agradecimentos - Ao CADCT/SEPANTEC-BA, PICDT/ Capes/UFBA, e ao CNPq pela bolsa e pelo auxílio financeiro concedido ao projeto de pesquisa; aos Professores Geraldo Carlos Pereira Pinto (Radam Brasil), Luciano Paganucci de Queiroz e Ana Maria Giulietti (HUEFS) e Maria Lenise Silva Guedes (UFBA), pela identificação das espécies vegetais; à equipe do Laboratório de Biologia e Ecologia de Abelhas (Labea), do IBUFBA, pela colaboração nos trabalhos de campo. Agradecemos ainda aos valiosos comentários e sugestões feitas pelos revisores dessa revista, que foram incorporadas à versão final do artigo.

\section{Referências bibliográficas}

ALBUQUERQUE, P. 1998. Abelhas silvestres (Hymenoptera, Apoidea) e suas fontes de alimento em um ecossistema de dunas, na ilha do Maranhão, Maranhão, Brasil: composição, fenologia e interações. Tese de doutorado, Universidade de São Paulo, Ribeirão Preto.

ALVES-DOS-SANTOS, I. 1999. Abelhas e plantas melíferas da mata atlântica, restinga e dunas do litoral norte do Estado do Rio Grande do Sul. Revista Brasileira de Entomologia 43:191-223.

ANDRADE, M.A.B. 1968. Contribuição ao conhecimento da ecologia das plantas das dunas do litoral do Estado de São Paulo. Boletim da Faculdade de Filosofia Ciências e Letras 305:3-170.
ARAÚJO, D.S.D. \& HENRIQUES, R.P.B. 1984. Análise florística das restingas do estado do Rio de Janeiro. In Restinga: origem, estrutura e processos (L.D. Lacerda, coord.). Comissão Editorial da Universidade Federal Fluminense, Rio de Janeiro, p.159-193.

ARAÚJO, D.S.D. \& LACERDA, L.D. 1987. A natureza das restingas. Ciência Hoje 33:42-48.

BAWA, K.S. 1980a. Breeding systems of trees in a tropical wet forest. New Zealand Journal of Botany 17:521-527.

BAWA, K.S. 1980b. Evolution of dioecy in flowering plants. Annual Review of Ecology and Systematics 11:15-39.

BAWA, K.S. 1983. Patterns of flowering in tropical plants. In Handbook of experimental pollination biology (C.E. Jones \& R.J. Little, eds.). Scientific and Academic Editions, New York, p. 394-410.

BRESSOLIN, A. 1979. Flora da restinga da ilha de Santa Catarina. Insula 10:1-54.

BRITTO, I.C., QUEIROZ, L P., GUEDES, M.L.S., OLIVEIRA, N.C. \& SILVA, L.B. 1993. Flora fanerogâmica das dunas e lagoas de Abaeté, Salvador, Bahia. Sitientibus 11:31-46.

CORDAZZO, C.V. \& SEELIGER, U. 1987. Composição e distribuição da vegetação das dunas costeiras ao sul de Rio Grande (RS). Ciência e Cultura 39:321-324.

CRONQUIST, A. 1981. An integrated system classification of flowering plants. Columbia University Press, New York.

FAEGRI, K. \& VAN DER PIJL, L. 1979. The principles of pollination ecology. Pergamon Press, Oxford.

FARIA, GM. 1994. A flora e a fauna apícola de um ecossistema de campo rupestre, Serra do Cipó - MG Brasil: composição, fenologia e suas interações. Tese de doutorado, Universidade Estadual Paulista, Rio Claro.

FURLAN, A., MONTEIRO, R., CESAR, O. \& TIMONI, J.L. 1990. Estudos florísticos das matas de restinga de Picinguaba, SP. In Anais do II Simpósio de Ecossistemas da costa sul e sudeste brasileira: estrutura, função e manejo (S. Watanabe, coord.). Aciesp, Águas de Lindóia, v.1, p. 220-227.

GOTTSBERGER, G, CAMARGO, J.M.F. \& SILBERBAUERGOTTSBERGER, I. 1988. A bee pollinated tropical community: The beach dune vegetation of Ilha de São Luís, Maranhão, Brazil. Botanische Jahrbücher für Systematik 109:469-500.

GREIG-SMITH, P. 1983. Quantitative plant ecology. Blackwell Scientific Publ., Oxford.

HEITHAUS, E.R. 1979. Flower visitation records and resource overlap of bees and wasps in Northwest Costa Rica. Brenesia 16:9-52.

KEVAN, P., GIURFA, M. \& CHITTKA, L. 1996. Why are there so many and few white flowers? Elsevier Science Ltd. 8:280-284.

LAROCA, S. 1974. Estudo feno-ecológico em Apoidea do litoral e primeiro planalto Paranaense. Dissertação de mestrado, Universidade Federal do Paraná, Curitiba.

LAROCA, S. 1995. Ecologia: princípios e métodos. Vozes, Petrópolis. 
LEWIS, GP. 1989. A new species of Poecilanthe (LeguminosaePapilionoideae) from Brazil. Kew Bulletin 44:167-169.

MAGNANINI,A. 1954. Contribuição ao estudo das zonas de vegetação de praia de Sernambetiba, DF. Arquivos Série Flora 8:147-232.

MARTINS, C.F. 1995. Flora apícola e nichos tróficos de abelhas (Hym., Apoidea) na Chapada Diamantina (Lençóis, BA, Brasil). Revista Nordestina de Biologia 10:119-140.

MORAWETZ, W. 1983. Dispersal and succession in an extreme tropical habitat: coastal sands and xeric woodland in Bahia (Brazil). Sonderband Naturwissenschaften Revue 7:359-380.

MORAWETZ, W. \& TILL, W. 1981. Aechemea itapoana, a new species from Bahia (Brazil): taxonomy and ecology. Plant Systematics and Evolution 138:147-151.

NEWSTROM, L.E., FRANKIE, GW., BAKER, H.G \& COLWELL, R.K. 1993. Diversity of long-term flowering patterns. In La Selva: Ecology and natural history of a Lowland Tropical Rainforest (L.A. McDade, K.S. Bawa, H.A Hespenheide \& GS. Hartshorn, eds.). University of Chicago Press, Chicago, p.142-160.

NEWSTROM, L.E., FRANKIE, GW. \& BAKER, H.G 1994. A new classification for plant phenology based on flowering patterns in lowland tropical rain forest trees at La Selva, Costa Rica. Biotropica 26:141-159.

PEDRO, S.R.M. 1992. Sobre as abelhas (Hymenoptera, Apoidea) em um ecossistema de cerrado (Cajuru, NE do Estado de São Paulo): composição, fenologia e visita às flores. Tese de doutorado, Universidade de São Paulo, Ribeirão Preto.

PEREIRA, O.J. \& GOMES, J.M.L. 1994. Levantamento florístico das comunidades vegetais de restinga no município de Conceição da Barra, ES. In Anais do III Simpósio de Ecossistemas da costa brasileira: subsídios a um gerenciamento ambiental: restingas e praias (S. Watanabe, coord.). Aciesp, Serra Negra, v.3, p.67-70.

PFADENHAUER, J. 1978. Contribuição ao conhecimento da vegetação e suas condições de crescimento nas dunas costeiras do Rio Grande do Sul. Revista Brasileira de Biologia 38:827-836.

PINTO, GL.P., BAUTISTA, H.P. \& FERREIRA, J.D.C.A. 1984. A restinga do litoral nordeste do estado da Bahia. In Restingas: origem, estrutura e processos (L.D. Lacerda, coord.).Comissão Editorial da Universidade Federal Fluminense, Rio de Janeiro, p.195-216.

PLOWMAN, T. 1983. New species of Erythroxylum from Brazil and Venezuela. Botanical Museum Leaflets 29:273-277.

POLHILL, R.M., RAVEN, P.H. \& STIRTON, C.H. 1981. Evolution and systematics of the Leguminosae. In Advances in Legume Systematics (R.M. Polhiil \& P.H. Raven, eds.). Royal Botanic Gardens, London.

PROCTOR, M., YEO,P. \& LACK, A. 1996. The natural history of pollination. Harper Collins Publishers, London.

RAWITSCHER, F.K. 1944. Algumas noções sobre a vegetação do litoral brasileiro. Boletim da Associação dos Geógrafos Brasileiros 5:13-28.
SAKAGAMI, S.F., LAROCA, S. \& MOURE, J.S. 1967. Wild bee biocenotics in São José dos Pinhais (PR), South Brazil. Preliminary report. Journal Faculty of Hokkaido University 19:190-250.

SCHWARTZ FILHO, D.L. 1993. A comunidade de abelhas silvestres (Hymenoptera, Apoidea) da Ilha das Cobras (Paraná, Brasil): aspectos ecológicos e biogeográficos. Dissertação de mestrado, Universidade Federal do Paraná, Curitiba.

SEABRA, J.J.A. 1949. A flora das dunas: apontamentos sobre a flora psamófila das dunas de Itapoã, Bahia. Lilloa 20:187-192.

SILVA, F.O., VIANA, B.F. \& JACOBI, C.M. 2005. The floral biology of Eriope blanchetii (Lamiaceae) in coastal sand dunes of NE Brazil. Austral Ecology 30:243-249.

SILVA, M.A. 1972. A flora das praias de Macéio. Anais do Instituto de Ciências Biológicas 2:11-121.

SILVA, M.C.M. 1998. Estrutura da comunidade de abelhas (Hymenoptera, Apoidea) de uma área de restinga (Praia de Intermares, Cabedelo - Paraíba, nordeste do Brasil). Dissertação de mestrado, Universidade Federal da Paraíba, João Pessoa.

SILVA, M.C.M. \& MARTINS, C.F. 1999. Flora apícola e relações tróficas de abelhas (Hymenoptera: Apoidea) em uma área de restinga (Praia de Intermares, CabedeloPB, Brasil). Principia 7:40-51.

SILVA, S.M., BRITEZ, R.M., SOUZA, W.S. \& JOLY, C.A. 1994. Fitossociologia do componente arbóreo da floresta de restinga da Ilha do Mel, Paranaguá, PR. In Anais do III Simpósio de Ecossistemas da costa brasileira: subsídios a um gerenciamento ambiental - restingas e praias ( $\mathrm{S}$. Watanabe, coord.). Aciesp, Serra Negra, v.3, p.33-48.

SOUTHWOOD, T.R.E. 1980. Ecological methods - with particular reference to the study of insect populations. Chapman and Hall, London.

SUGIYAMA, M. \& MANTOVANI, W. 1994. Fitossociologia de um trecho de mata de Restinga na Ilha do Cardoso, SP. In Anais do III Simpósio de Ecossistemas da costa brasileira: subsídios a um gerenciamento ambientalrestingas e praias (S. Watanabe, coord.). Aciesp, Serra Negra, v.3, p. 49-57.

TORREND, C.S.J. 1938. Nova Contribuição para a Flora da Bahia. In Separata do Anuário da Escola Agrícola da Bahia, Salvador, Brasil, p. 258-330.

VIANA, B.F., KLEINERT, A.M.P., SILVA, F.O. 2002. Ecologia de Xylocopa (Neoxylocopa) cearensis (Hymenoptera, Anthophoridae) nas Dunas Litorâneas de Abaeté, Salvador, Bahia. Iheringia 92:47-57.

VOGEL, S. 1969. Flowers offering fatty oil instead of nectar. In Abstracts $11^{\text {th }}$ International Botanical Congress, Seatle, p.229.

WALTER, H. 1963. The water relation of plants, London. Blackwell Scientific Publications.

ZANELLA, F.C.V. 1991. Estrutura da comunidade de abelhas silvestres (Hymenoptera, Apoidea) da Ilha do Mel, planície litorânea Paranaense, sul do Brasil. Dissertação de mestrado, Universidade Federal do Paraná, Curitiba. 
